\title{
əCoordinated Global and Regional Climate Modeling*
}

\author{
J. F. Scinocca, V. V. Kharin, Y. JiAO, M. W. Qian, M. LAZARE, \\ L. SOLHEIM, AND G. M. FlATO \\ Canadian Centre for Climate Modelling and Analysis, Environment Canada, Victoria, \\ British Columbia, Canada
}

\author{
S. BINER \\ Consortium Ouranos, Montreal, Quebec, Canada
}

\author{
M. Desgagne And B. Dugas
}

Division de Recherche en Prévision Numérique, Environment Canada, Montreal, Quebec, Canada

(Manuscript received 28 February 2015, in final form 23 July 2015)

\begin{abstract}
A new approach of coordinated global and regional climate modeling is presented. It is applied to the Canadian Centre for Climate Modelling and Analysis Regional Climate Model (CanRCM4) and its parent global climate model CanESM2. CanRCM4 was developed specifically to downscale climate predictions and climate projections made by its parent global model. The close association of a regional climate model (RCM) with a parent global climate model (GCM) offers novel avenues of model development and application that are not typically available to independent regional climate modeling centers. For example, when CanRCM4 is driven by its parent model, driving information for all of its prognostic variables is available (including aerosols and chemical species), significantly improving the quality of their simulation. Additionally, CanRCM4 can be driven by its parent model for all downscaling applications by employing a spectral nudging procedure in CanESM2 designed to constrain its evolution to follow any large-scale driving data. Coordination offers benefit to the development of physical parameterizations and provides an objective means to evaluate the scalability of such parameterizations across a range of spatial resolutions. Finally, coordinating regional and global modeling efforts helps to highlight the importance of assessing RCMs' value added relative to their driving global models. As a first step in this direction, a framework for identifying appreciable differences in RCM versus GCM climate change results is proposed and applied to CanRCM4 and CanESM2.
\end{abstract}

\section{Introduction}

At the Canadian Centre for Climate Modelling and Analysis (CCCma), a new regional climate model, the CCCma Regional Climate Model (CanRCM4), has

¿ Denotes Open Access content.

* Supplemental information related to this paper is available at the Journals Online website: http://dx.doi.org/10.1175/JCLI-D-15-0161.s1.

Corresponding author address: J. F. Scinocca, Canadian Centre for Climate Modelling and Analysis, Environment Canada, P.O. Box 3065 STN CSC, Victoria, BC V8W 3V6, Canada. E-mail: john.scinocca@ec.gc.ca been developed. CanRCM4's novelty does not arise from the method of solution in its dynamical core or the climate-based physics package it employs. Both of these are well known and currently operational for global model applications. The novelty of CanRCM4 stems from a new philosophy of coordinating the development and application of regional climate models (RCMs) and global climate models (GCMs). It is argued here that the development and application of an RCM that is tightly connected to a global parent model offers new and unique opportunities that are not accessible to independent regional climate modeling efforts. We shall refer to this approach as a "coordinated modeling" framework, and it involves a strict adherence to physics compatibility between the RCM and driving GCM. The application of spectral nudging (Biner et al. 2000; von Storch et al. 
2000; Miguez-Macho et al. 2004) in both the regional and parent global model is also an important feature of this new approach.

Following a strategy of "strict physics compatibility," CanRCM4 shares exactly the same package of physical parameterizations with CCCma's global atmospheric climate model (CanAM4; von Salzen et al. 2013). Strict physics compatibility offers a number of advantages for both global and regional climate modeling. It allows a direct investigation of the scalability of parameterizations (i.e., the ability to operate physically over a range of spatial scales). Parameterizations require some assumptions about the subgrid structure of their input fields, including the effects of intermittency. This is often characterized by a probability density function (PDF) of the subgrid structure. Through carefully constructed pairs of GCM-RCM simulations, the properties of such subgrid PDFs for a given GCM can be informed by companion RCM simulations. Such a program of strict physics compatibility would, therefore, facilitate the development of improved GCM parameterizations and provide a systematic method to investigate their scalability down to RCM resolutions. If it were the case that, for a particular physical process, strict compatibility did not work well, such a coordinated approach would help provide explicit reasons why. By sharing the same physics package, and pursuing the study of scalability in this way, CanRCM4 enjoys the benefits of the physics compatibility approach discussed by Giorgi and Mearns (1999) while maintaining a line of model development that addresses the main potential disadvantage of this approach, which was identified as "physics adequacy" in that study.

An additional advantage of strict physics compatibility is that it leads to improved RCM driving at domain boundaries. Climate model physics packages have undergone a dramatic increase in complexity over the last decade with the introduction of prognostic cloud schemes, aerosolcloud interactions, and atmospheric chemistry. The increasing number and variety of prognostic variables used to represent such physical processes differ markedly across models and generally will not match between an RCM and the GCM that provides its driving data. Consequently, RCMs are generally limited to dynamical driving (i.e., winds, temperature, and moisture) on their lateral boundaries. As a result, within an RCM, it is not possible to represent the distribution and evolution of aerosols and chemical species that have source regions outside the RCM domain. For example, this would be particularly problematic for an RCM study of Arctic air quality where, for the most part, pollutants such as sulfate aerosol have their sources well outside the Arctic and undergo long-range transport into this region. By employing a strategy of strict physics compatibility, all prognostic aerosol and chemical species represented in the driving model can be prescribed on the RCM's lateral boundaries, allowing an all-field driving and, therefore, consistent representation and downscaling of species transported into the RCM domain.

By the inclusion of spectral nudging in CanRCM4's parent global model, CanESM2, such all-field driving of the RCM can also been extended to the case when CanRCM4 is driven by either reanalysis data or another GCM. This is accomplished by a two-step procedure. In the first step, the winds, temperatures, and specific humidity in the global model are constrained by a spectral nudging procedure to follow the evolution described by the reanalysis or external GCM. In this way, all other prognostic fields related to aerosols and chemistry in the global model are naturally slaved to this evolution and so made consistent with the foreign driving data. In the second step, the output of this constrained global simulation is used to provide an allfield driving of the RCM. The ability to derive such a complete set of driving fields requires both a parent global model and a philosophy of strict physics compatibility. In principle, therefore, all-field driving of CanRCM4 can be employed for all of its applications.

Finally, in employing a coordinated modeling framework, the question of value added by the RCM becomes a central issue. By construction, any future prediction or projection made by an RCM designed to address a question about the climate system must be preceded by a GCM prediction or projection designed to address the identical question. In other words, prior to any RCM simulations, an understanding of that issue will already be available from the global model. From this perspective, the RCM downscaling exercise is one of adding understanding (or value) to an existing global model result. A global climate modeling center that diverts resources toward a program of regional climate modeling must ultimately justify the use of those resources. Such justification is inextricably connected to the question of value added by the RCM.

The outline of this paper is as follows. In section 2, we provide a new perspective on the properties of the oneway nesting methodology to better understand the potential value, and artifacts, associated with the use of spectral nudging. In section 3, a summary of CanRCM4 is provided, including the details of the spectral nudging procedures that are used to coordinate the global and regional simulations. In section 4 , a series of sensitivity experiments is presented that includes CanRCM4 driven directly by reanalysis compared to CanRCM4 driven in an equivalent fashion by its parent model following the two-step procedure designed to constrain the global model to follow reanalysis. Additionally, the impact of all-field driving versus dynamical-field driving is presented. In section 5, a framework is presented that 
represents a first step toward the assessment of value added by RCM downscaling relative to its global driving models. Finally, in section 6 , we conclude with a brief summary of the results of this study.

\section{The one-way nesting methodology}

The development and application of global and regional climate models are complex exercises. While the two efforts share many similarities, the one-way nesting methodology typically used by RCMs presents a set of additional challenges that are unique to its experimental design. At its core, one-way nesting is a deterministic modeling approach applied to an inherently chaotic system. That is, an RCM's prognostic variables are required to follow a specified time-evolving realization of synopticscale evolution, or weather, on its lateral boundaries but it is also expected to deviate from that realization in its interior. In principle, this represents an inconsistency that can lead to artifacts not present in global models or in the real atmosphere. Of all the complexities associated with regional climate modeling, those with their roots in this inconsistency are most concisely explained by two intractable issues, which we shall refer to as "chaotic divergence" and "upscale influence."

Chaotic divergence arises from the fundamental nonlinearity that is responsible for the well-known 2-week predictability limit of numerical weather prediction. Because of such nonlinearity, the realization of weather (i.e., synoptic and larger scales) within the RCM must deviate from that imposed on its lateral boundaries. The extent of this deviation at any particular time and location is strongly influenced by the residency time of air parcels within the RCM domain (Lucas-Picher et al. 2008a). The residency time of an air parcel, defined as the period between it being introduced into and then flushed out of the RCM domain, roughly determines the time available for nonlinearity to modify the realization. The longer the residency time, the longer nonlinearity is able to act and the larger the deviation from the driving data. Such deviation is often referred to as a "lack of control" exerted by the lateral boundary conditions (Laprise 2008), and it contributes to the internal variability of the RCM solution (Lucas-Picher et al. 2008b; Alexandru et al. 2009). Here, we shall take chaotic divergence to refer to the magnitude of deviation that is induced by such nonlinearity and is limited by air parcel residency time in the RCM.

Chaotic divergence is a function of the size and geophysical properties of the domain, the seasonal and transient flow conditions, and the specific properties of the RCM. It is problematic because the decoupling of the internal realization of weather from that imposed on the lateral boundaries (Kanamaru and
Kanamitsu 2007) is artificial and could potentially lead to unphysical influence on the RCM results. Such potential artifacts include a loss of nonlocal influence (e.g., teleconnections initiated outside the RCM domain), a restriction of the largest spatial scale downscaled by the RCM to that which spans the RCM domain, and any influence from the complicated fluid motions in the time-evolving decoupling zone over which the prescribed weather on the boundary transitions into internally generated weather in the RCM interior. Such potential artifacts will be highly variable in space and time and so difficult to quantify.

Upscale influence, on the other hand, arises from the disparity of spatial scales between the driving data and that simulated by the RCM. For the example of GCM driving data, the higher-resolution RCM will simulate features and processes that were absent or poorly resolved in the GCM. In general, such additional dynamics will have a systematic feedback on the large scales in the RCM. Such feedback is physically realistic and may be interpreted as acting systematically to correct those biases in the GCM climate that arise from the absence of such fine scales. However, as such influence intermittently moves upscale in the RCM, it promotes chaotic divergence, enhancing inconsistencies, or decoupling, between the realization of weather imposed on its lateral boundaries and that which develops in its interior. Upscale influence is anticipated to be most problematic for RCM applications that are driven on their lateral boundaries by model (GCMs) rather than reanalysis or other observationally constrained data [i.e., dynamical downscaling types 3 and 4 rather than types 1 and 2, as defined by Castro et al. (2005)]. While upscale influence can exacerbate the level of chaotic divergence discussed above, the two effects are distinct. For example, if spatial resolutions of the RCM and driving GCM are identical, upscale influence is absent while chaotic divergence remains an issue (e.g., Koltzow et al. 2008).

The ideal goals would be to minimize chaotic divergence and allow upscale influence to systematically act on any correctable biases in the large-scale driving flow. However, these goals act at cross purposes and in general cannot be satisfied simultaneously for even the most carefully constructed RCM simulation. Over the past decade, the RCM community has explored additional strategies, such as spectral nudging (Biner et al. 2000; von Storch et al. 2000; Miguez-Macho et al. 2004), to constrain the large-scale RCM flow to follow the driving data within its domain. Spectral nudging provides a very powerful and direct means to control chaotic divergence. Its use, however, has the side effect of inhibiting upscale influence from systematically acting on any correctable biases in the large-scale driving flow. From this perspective, the RCM community's philosophical debate 
regarding the appropriateness of spectral nudging for RCM studies (e.g., see Bowden et al. 2013) may be seen more as a debate about the appropriateness of allowing systematic upscale influence on the driving flow.

Following these ideas, there does seem to be one RCM application where the conflict described above is not an issue: when an RCM is driven by reanalysis data and a procedure, such as spectral nudging, is used to control chaotic divergence. In principle, reanalysis data contain little or no correctable bias because their synoptic scales are well resolved in observations and have felt the true influence of all spatial scales. In this instance it is not necessary, or desirable, to allow systematic upscale influence in the RCM, as such influence would serve only to degrade the flow being downscaled. Consequently, the basic concepts presented here would predict that downscaling in reanalysis-driven RCM experiments would be most successful when a procedure like spectral nudging is included. In practice, the application of spectral nudging in reanalysis-driven RCM simulations has been overwhelmingly found to provide the most credible results (Miguez-Macho et al. 2004; Rockel et al. 2008; Tang et al. 2010; Song et al. 2011; Feser and Barcikowska 2012; Otte et al. 2012; Bowden et al. 2013; Glisan et al. 2013). From this perspective, it is arguable that an evaluation of the relative downscaling ability of each RCM in the next phase of the Coordinated Regional Climate Downscaling Experiment (CORDEX; Giorgi et al. 2009) would benefit from an additional reanalysis-driven evaluation simulation that included spectral nudging.

The difficulty arises when the RCM driving data is derived from a freely running GCM rather than observations, as is required when an RCM is used to investigate future climate change issues. In this situation, the large scales of the driving data arguably contain some amount of "correctable bias," and the benefit of allowing upscale influence becomes an open question. There are essentially two options: employ a procedure like spectral nudging to control chaotic divergence, which inhibits upscale influence; or employ no procedure to control chaotic divergence, which allows upstream influence to systematically alter the large scales. If one chooses the former option, correctable biases in the GCM driving data will remain and potentially impact the dynamical downscaling produced by the RCM (Giorgi 2006). If one chooses the latter option, upscale influence can act on correctable GCM biases, but it will also exacerbate chaotic divergence, leading to large-scale inconsistencies and decoupling between the RCM solution and GCM driving data, potentially leading to artifacts in the downscaling results produced by the RCM.

Currently, it is not clear which of these two options offers the least amount of artifacts or whether the presence of such artifacts would influence the results of an RCM study of climate change. What is clear is that, in principle, chaotic divergence and upscale influence are the source of artifacts in the one-way nesting procedure that can never be completely eliminated from even the most carefully designed and implemented RCM studies of climate change. As such, it is important to distinguish them from the variety of more tractable issues that may also introduce artifacts into downscaling studies of climate change. Such issues include physics compatibility between driving and downscaling models (Giorgi and Mearns 1999), completeness of RCM driving data, and regionspecific tuning of RCM physics (Takle et al. 2007; Jacob et al. 2012). Artifacts arising from these issues are just as difficult to characterize and quantify, but they can, in principle, be controlled or eliminated from RCM simulations. In this study, we describe the development and application of a new regional climate model where we have paid particular attention to such controllable issues.

\section{Coordinated modeling setup}

The current version of the CCCma global earth system model (CanESM2; Arora et al. 2011) employs a fourthgeneration atmospheric physics package, which is the product of a multidecadal program of climate model development at the CCCma. The full atmospheric model, CanAM4, employs a spectral dynamical core and so offers no straightforward path to limited-area integrations. To facilitate an ongoing program of regional climate modeling, it was decided to transfer the full physics package from CanAM4 to the limited-area configuration of the Global Environmental Multiscale (GEM) model (Côté et al. 1998), which was developed by Environment Canada's Recherche en Prévision Numérique (RPN) and employed for both global and regional numerical weather prediction. Transfer of the complete CCCma physics package to this new dynamical core allows both global model (GEM) and limited-area model (GEM-LAM) configured integrations to be performed. The global configuration is particularly useful to validate the rather complex exercise of transplanting the complete physics package into GEM-LAM, and this will be discussed later in this section.

The new regional climate modeling effort discussed here is distinct from another well-established regional climate modeling program in Canada, and it is useful to distinguish the two. The Canadian Regional Climate Model (CRCM) is currently on its fifth generation and is the culmination of coordinated government and university efforts initiated in 1991 at the University of Quebec at Montreal. An excellent summary of CRCM5 and its history of development is provided by Zadra et al. (2008). The development of CRCM5 involved the 
move to using GEM-LAM as its dynamical core based on a need for more efficient computation. In its most recent applications, CRCM5 employs physical parameterizations composed of a selection from the GEM "meso-global" libraries developed by RPN for numerical weather prediction applications (Hernandez-Diaz et al. 2012). CRCM5 is thus an example of a collaborative effort between a modern global operational forecast provider and a university-based regional climate research initiative. While both CanRCM4 and CRCM5 employ the same dynamical core (GEM-LAM), the independence of their physics packages means that each model represents a very distinct RCM. Furthermore, CanRCM4 is developed and exercised within a program of coordinated global and regional climate modeling, while CRCM5 is more representative of an independent RCM development and application effort. In this section, we summarize the various components of CanRCM4, validate its operation under the new dynamical core, and review the algorithms employed to nudge the GCM and RCM in our coordinated modeling framework.

\section{a. Model description}

The fourth-generation atmospheric physics package employed by CanESM2 and CanRCM4 was recently used for the Canadian contribution to phase 5 of the Coupled Model Intercomparison Project (CMIP5). It is described in its entirety by von Salzen et al. (2013), and it represents a significant advancement over the thirdgeneration package presented by Scinocca et al. (2008). For example, the radiative transfer scheme has been replaced by a new scheme that includes a correlated- $k$ distribution model (Li 2002; Li and Barker 2005) and a more general treatment of radiative transfer in cloudy environments using the Monte Carlo independent column approximation (McICA) methodology (Pincus et al. 2003; Barker et al. 2008). Coupled with other parameterizations, the radiative transfer scheme accounts for the direct and indirect radiative effects of aerosols. A prognostic bulk aerosol scheme with a full sulfur cycle, along with organic and black carbon, mineral dust, and sea salt has been added (Lohmann et al. 1999; von Salzen et al. 2000; Croft et al. 2005). A fully prognostic single-moment cloud microphysics scheme is now used in the model, based on the work of Lohmann and Roeckner (1996), Rotstayn (1997), and Khairoutdinov and Kogan (2000). A statistical approach is used for macrophysical properties of layer clouds (Chaboureau and Bechtold 2005), and a new shallow convection scheme has been added to the model (von Salzen et al. 2005). Approaches to local and nonlocal turbulent mixing by Abdella and McFarlane (1996) and McFarlane et al. (2006) were also improved in this version.
The GEM model (Côté et al. 1998) and GEM-LAM are described by Zadra et al. (2008). They were developed for global and regional numerical weather prediction by Environment Canada's Canadian Meteorological Centre. GEM is a two-time-level semiLagrangian model. While a fully elastic nonhydrostatic configuration of GEM has been implemented (Yeh et al. 2002), the configuration employed here for CanRCM4 is hydrostatic and employs a hybrid pressureand terrain-following vertical coordinate and a regular latitude-longitude grid with rotated pole. To facilitate its transfer to the GEM dynamical core, the CCCma physics package in its entirety was localized under one driver routine and modified to eliminate any dependence on the algorithm, numerics, and time stepping employed by the dynamical core. At the same time, a similar exercise was undertaken in the GEM model development to ensure that the dynamical core was independent of the particular physics package called during its execution. A procedure was then implemented so that the physics code could be called by the CCCma global spectral model and the GEM model. While this represented a substantial multiyear technical effort, it was designed in such a way to ensure that it would not have to be repeated for the transfer of newer versions of the CCCma physics package. Validation is provided in the next subsection, in which 5-yr climatologies of CanAM4 and GEM employing the fourthgeneration CCCma physics package are compared.

\section{b. Validation of physics package porting}

The porting and installation of CanAM4 physics into the GEM3.3 dynamical core was first tested in a global climate configuration. We shall refer to this configuration as CanAM $4^{\mathrm{GEM}}$. Assuming that both the spectral and semiLagrangian dynamical cores are adequate to similarly represent the leading-order effects of advection, one would expect similar climatologies from each model when the same physics package is employed.

To facilitate this comparison, similar spatial resolutions were employed for each model. Since CanAM4 is typically run at a triangular spectral truncation of T63, the corresponding linear Gaussian grid of 128 longitudes and 64 latitudes was selected for the CanAM4 ${ }^{\mathrm{GEM}}$ simulation. Both models employed a vertical domain with a lid near $1 \mathrm{hPa}$. The semi-Lagrangian algorithm in GEM3.3 was not formulated to be conservative for mass and momentum (Côté et al. 1998). While this is not an issue for numerical weather prediction, or for the use of GEM-LAM as an RCM, it can cause drift over longer time scales for global climate simulations. Therefore, for the climate comparison of CanAM4 versus CanAM4 ${ }^{\mathrm{GEM}}$, only short climate simulations were performed. A 5-yr AMIP-type 
Total Precipitation

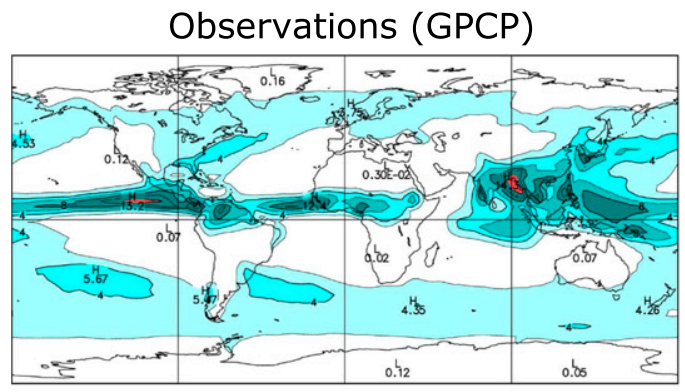

CanAM4

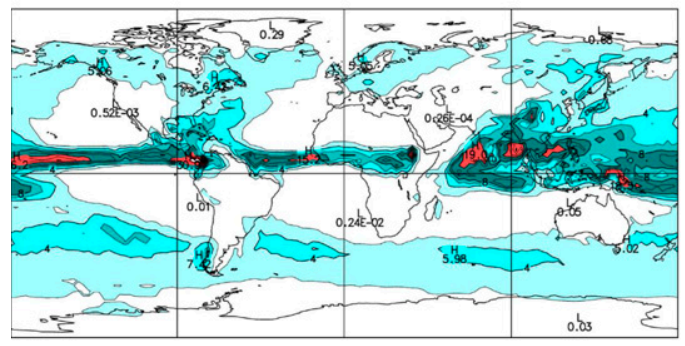

GEM (CanAM4 physics)

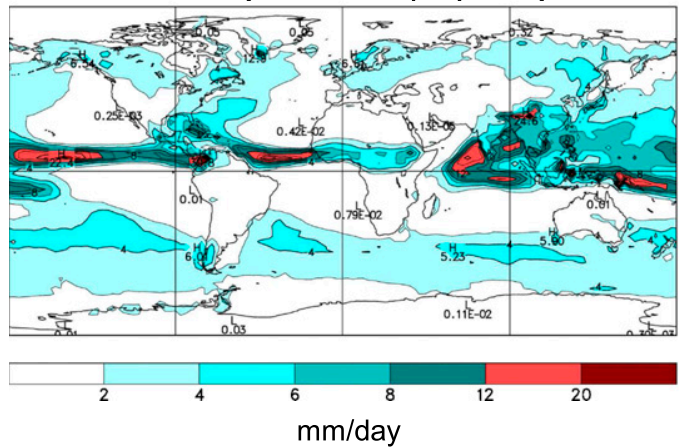

Total Cloudiness

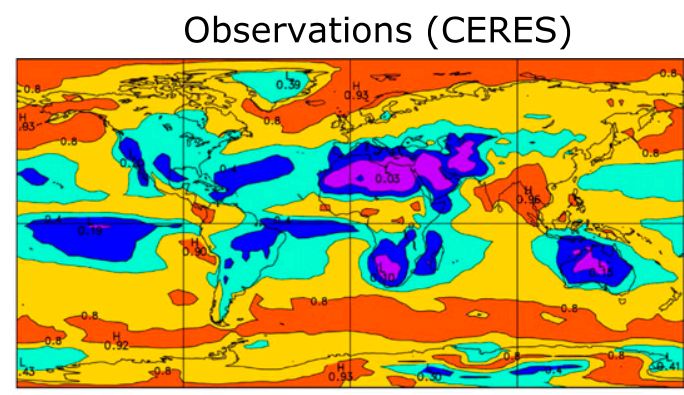

CanAM4

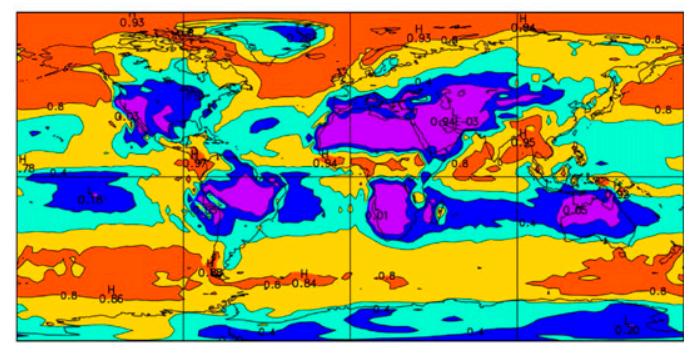

GEM (CanAM4 physics)

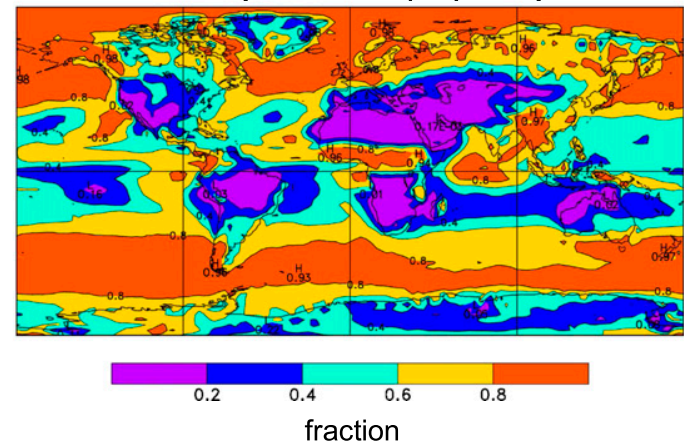

FIG. 1. Comparison of CanAM4 vs global configuration CanAM4 ${ }^{\text {GEM }}$ (i.e., GEM3.3 employing the CanAM4 physics package). (left) JJA total precipitation and (right) JJA total cloudiness from (top) observations [precipitation derived from the Global Precipitation Climatology Project, version 2 (Adler et al. 2003), and cloudiness derived from the Clouds and the Earth's Radiant Energy System project (Wielicki et al. 1996; Minnis et al. 2003)]; (middle) CanAM4; and (bottom) CanAM4 ${ }^{\mathrm{GEM}}$.

simulation corresponding to the years $1978-82$ was undertaken, with each model employing the corresponding external forcings of greenhouse gas concentrations, aerosol emissions, and sea surface temperatures (SSTs), including sea ice distribution employed for the CMIP5 AMIP simulations. This 5-yr period was sufficient to define representative climatologies for a variety of atmospheric quantities but not so long that drift as a result of a lack of conservation in GEM3.3 would significantly impact the comparison.

Figure 1 presents 5-yr climatologies of June-August (JJA) total precipitation and total cloudiness from observations and from CanAM4 and CanAM4 ${ }^{\text {GEM }}$. These fields represent prognostic quantities that strongly depend on the inherent properties of the physics package. It is apparent from this comparison that, for both precipitation and cloudiness, CanAM4 and CanAM4 ${ }^{\mathrm{GEM}}$ provide strikingly similar climatological distributions, which have similar biases relative to the observations. Such agreement suggests that the physics packages are operating similarly in each model and provides confidence that the CanAM4 physics package has been correctly ported to the GEM model.

\section{c. CanRCM4 configuration details}

CanRCM4 employs the limited-area horizontal grid configuration of the GEM3.3 model. Strict physics 
compatibility means that CanRCM4 and its parent CanAM4 version employ exactly the same physics package (von Salzen et al. 2013). The CanAM4 physics package has a large number of free parameters that are typically adjusted when it is employed at different spatial resolutions. Such readjustment of the free parameters is an involved exercise and should likely employ additional criteria for optimal downscaling performance in the RCM. At present, such a retuning of CanRCM4 has not been performed. Consequently, in addition to using a global model physics package, in its first implementation CanRCM4 also uses the same free parameter settings as the global model for all of its physical parameterizations. The expectation then is that the implementation of CanRCM4 discussed in this study should be at a disadvantage relative to more mature regional climate models. In the supplemental material, CanRCM4's performance is compared against the performance of RCMs that participated in the North American Regional Climate Change Assessment Program (NARCCAP) (Mearns et al. 2009). Following the NARCCAP experiment protocols and repeating diagnostic analyses performed in Mearns et al. (2012), it is found that CanRCM4's performance falls within that of the RCMs analyzed in Mearns et al. (2012). These results provided confidence that this configuration of CanRCM4 could be used for the CORDEX used to downscale global climate model contributions to CMIP5. Additional details follow in this section.

Unless otherwise stated, CanRCM4 employs specified sea surface temperature and sea ice distributions that match those in its parent global model and has a model lid height of $13 \mathrm{hPa}$, employing essentially the same model levels as its parent model below this elevation. CanRCM4 contributed simulations for four regional domains to CORDEX: North America, the Arctic, Africa, and Europe. For each of these domains, the following suite of CORDEX experiments were performed: evaluation (1989-2009), for which driving data is provided by the $1.5^{\circ} \times 1.5^{\circ}$ resolution ERA-Interim dataset (Dee et al. 2011); historical (1950-2005); and two future scenarios (2006-2100) with representative concentration pathways (RCPs) 4.5 (RCP4.5) and 8.5 (RCP8.5). Driving data for the historical and future RCP simulations of CanRCM4 were provided by ensemble member 1 of CanESM2's contribution to CMIP5. This suite of CanRCM4 CORDEX experiments was performed for horizontal spatial resolutions of $0.44^{\circ}(50 \mathrm{~km})$ and $0.22^{\circ}(25 \mathrm{~km})$. CanRCM4 output from these runs is available from the Earth System Grid Federation (www. earthsystemgrid.org) or directly from the CCCma website (www.cccma.ec.gc.ca/data/canrcm/CanRCM4).
Assimilation of the driving data at the lateral boundaries of CanRCM4 is facilitated by a blending region around the lateral perimeter of the RCM domain where the RCM solution is nudged toward the driving data (e.g., Davies 1976). The formulation of the nudging employed in this blending region of GEMLAM was modified for CanRCM4 applications to use a relaxation time scale and dimensional width. This formulation facilitated the specification of identical blending regions (both in spatial scale and strength) for RCM simulations employing the same domain but different spatial resolutions. Under this formulation, minimum and maximum time scales are respectively set for the outermost and innermost edges of the blending region. A blending region of width $4.4^{\circ}$ or roughly $500 \mathrm{~km}$ is employed around the perimeter of the RCM domain for all applications. In this region, a relaxational tendency of the form

$$
\left(\frac{d X}{d t}\right)_{\text {blend }}=-\frac{1}{\tau_{b}}\left(X-X_{\text {drive }}\right)
$$

is applied on the RCM grid, where $X$ is a prognostic variable of the RCM that is driven by the external driving data $X_{\text {drive }}$, and $\tau_{b}$ is the relaxation time scale. The time scale $\tau_{b}$ increases from a value of $1 \mathrm{~h}$ at the outermost edge of the blending region to $24 \mathrm{~h}$ at the innermost edge of the blending region following a squared sine profile over the range from 0 to $\pi / 2$.

As discussed earlier, spectral nudging of the atmospheric component of CanRCM4's parent model, CanESM2, is employed to facilitate all-field driving of CanRCM4. For this purpose, relaxational spectral nudging of the form

$$
\left(\frac{d X}{d t}\right)_{s}^{\mathrm{GCM}}=-\frac{1}{\tau_{s}} \Gamma_{n}^{\mathrm{SH}}\left(X-X_{\text {ref }}\right)
$$

is applied in spectral space, where $\tau_{s}$ is the relaxational time scale, and the operator $\Gamma_{n}^{\mathrm{SH}}(\cdot)$ is a spectral filter employing a triangular cutoff at total wavenumber $n$ of a spherical harmonic spatial representation of the nudged fields. This form of relaxational nudging was recently used in the empirical bias-correction study of Kharin and Scinocca (2012). The nudging (2) is integral to the two-step procedure employed to provide all-field driving of CanRCM4 for experiments when the source of driving data is not its parent global model. In the first step, spectral relaxation (2) is employed to nudge the global model toward the reference driving data (e.g., ERA-Interim data for the CORDEX evaluation simulations), which is typically available with a 6-h update frequency. The winds, temperature, and specific 
humidity are nudged with a time scale of $\tau_{s}=24 \mathrm{~h}$ and T21 spectral truncation on all model levels. In the second step, the parent model is then used to supply lateral boundary driving data for CanRCM4 simulations at 6-h frequency for all prognostic variables.

The spectral nudging employed in CanRCM4 is of the form

$$
\left(\frac{d X}{d t}\right)_{s}^{\mathrm{RCM}}=-\frac{1}{\tau_{s}} \Gamma_{n}^{\mathrm{DC}}\left(X-X_{\text {drive }}\right),
$$

where the operator $\Gamma_{n}^{\mathrm{DC}}(\cdot)$ is a low-pass spectral filter based on a discrete cosine transform (Denis et al. 2002) and applied on the RCM grid. Following the properties of spectral nudging employed for the parent global model, a time scale of $\tau_{s}=24 \mathrm{~h}$ and a spectral filter with a cutoff approximating T21 spectral truncation is employed in CanRCM4. The spectral transfer function of this filter is comprised of a Gaussian profile with half height corresponding to a cutoff spatial scale of $410 \mathrm{~km}$, which roughly corresponds to T21 spectral truncation of global fields. All driving or reference fields in (1)-(3) are updated every $6 \mathrm{~h}$ and interpolated linearly down to the time step of the model.

Following the approach of early studies (von Storch et al. 2000; Biner et al. 2000), most implementations of spectral nudging operate predominantly in the upper troposphere and lower stratosphere of RCMs (e.g., Miguez-Macho et al. 2004; Alexandru et al. 2009; Glisan et al. 2013). The apparent motivation for restricting the nudging to these higher elevations is to strike a balance between controlling large-scale biases in the RCM and allowing the RCM solution to develop freely in the lower troposphere. Here, a deeper vertical structure of spectral nudging is employed to better control the occurrence of chaotic divergence as discussed in section 2. In CanRCM4, a constant spectral nudging is applied to the horizontal wind components from the model top down to $850 \mathrm{hPa}$. Below this level, the inverse of the relaxation time scale $\left(1 / \tau_{s}\right)$ is smoothly decreased toward zero at the surface following a squared cosine profile over the range from 0 to $\pi / 2$. Spectral nudging is also applied to the temperature field on the top three vertical levels of the model domain employing the same properties as that for the winds in this region. This combination of spectral nudging on wind and temperature at the top of the model was found to provide a good open boundary condition for upwardly propagating planetary waves.

\section{Driving sensitivity experiments}

In this section, CanRCM4 simulations driven by the reanalysis data directly are compared to corresponding simulations driven by its parent global model constrained to follow the reanalysis. Simulations discussed in this section follow the CORDEX specifications for the evaluation experiment over the North American domain (19892009). All simulations employ the regional spectral nudging (3) defined in section 3c. Three simulations are intercompared. The first is driven directly by winds (zonal and meridional), temperature, and specific humidity $(U, V, T$, and $q$, respectively) from the ERA-Interim. The other two simulations are driven by the output of CanRCM4's parent global model from a simulation in which it was nudged toward ERA-Interim following the global spectral nudging prescription [(2) in section 3c]. The second simulation of CanRCM4 is driven by the same fields as in the first simulation (i.e., by $U, V, T$, and $q$ only). In the third simulation all prognostic variables of CanRCM4 are driven on its lateral boundaries (all-field driving).

It is anticipated that all-field driving will be most important for prognostic variables, such as aerosols, which are model specific and so generally not available for RCM driving. Figures 2 and 3 present the mean JJA distribution of mineral dust and sulfate aerosol, respectively, from these three simulations in Figs. $2 \mathrm{a}-\mathrm{c}$ and $3 \mathrm{a}-\mathrm{c}$. Figures $2 \mathrm{~d}-\mathrm{f}$ and $3 \mathrm{~d}-\mathrm{f}$ show differences between CanRCM4 runs and CanRCM4's nudged parent run. A comparison of the ERA-Interimdriven simulation (Figs. 2a and 3a) with that driven by the nudged parent model shows little difference when driven with the same variables $(U, V, T$, and $q)$ on its lateral boundaries (Figs. 2b and 3b). Their similarity is evidence that the nudged parent model is a good proxy for the ERA-Interim dynamical and thermodynamic driving of the RCM.

When all-field driving of CanRCM4 is employed (Figs. 2c and 3c), there are significant differences in the simulation of these two tracers. For mineral dust (Fig. 2), a broad region of elevated concentrations in the southeast quadrant of the domain is apparent, which is completely absent in simulations that do not include mineral dust as a boundary forcing (Figs. 2a,b and associated difference plots in Figs. 2d,e). The origin of this dust is the African Sahara, with Atlantic transit times of roughly 7-10 days (Engelstaedter et al. 2006). Such dust serves as a host for microorganisms and has been implicated as a risk to human health (Griffin 2007). For sulfate aerosol, $\mathrm{SO}_{4}$ (Fig. 3), even though there are significant North American sources, it is clear that the background value is underestimated when sources such as Asia are excluded by not driving $\mathrm{SO}_{4}$ on the lateral boundaries of the RCM.

The impact of future climate change on air quality and health in any region will generally depend on changing aerosol sources and global circulation patterns outside of that 
ERA-interim Driven $(\mathbf{U}, \mathbf{V}, \mathbf{T}, \mathbf{q})$

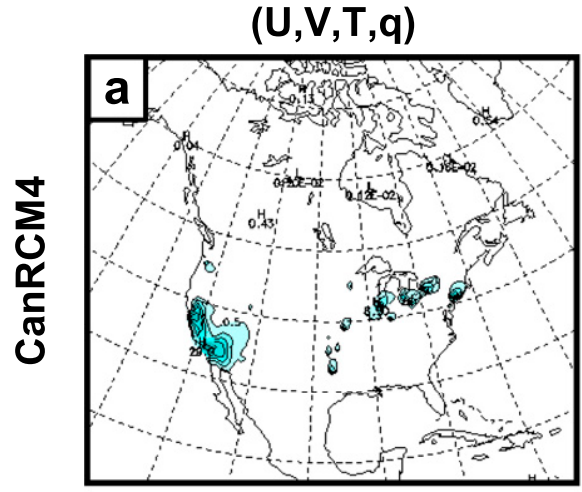

Nudged Parent Driven (U,V,T,q only)
Nudged Parent Driven (full-field)
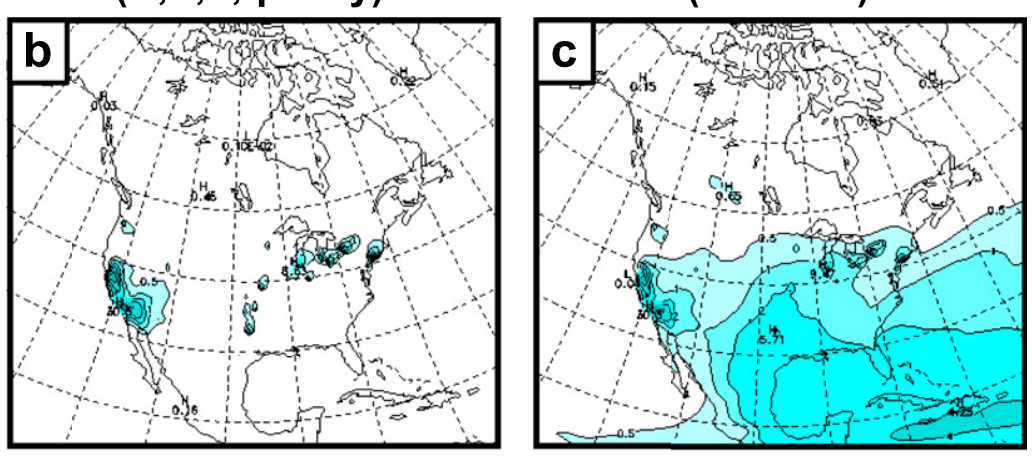

$\operatorname{ppbw}(\times 0.1)$

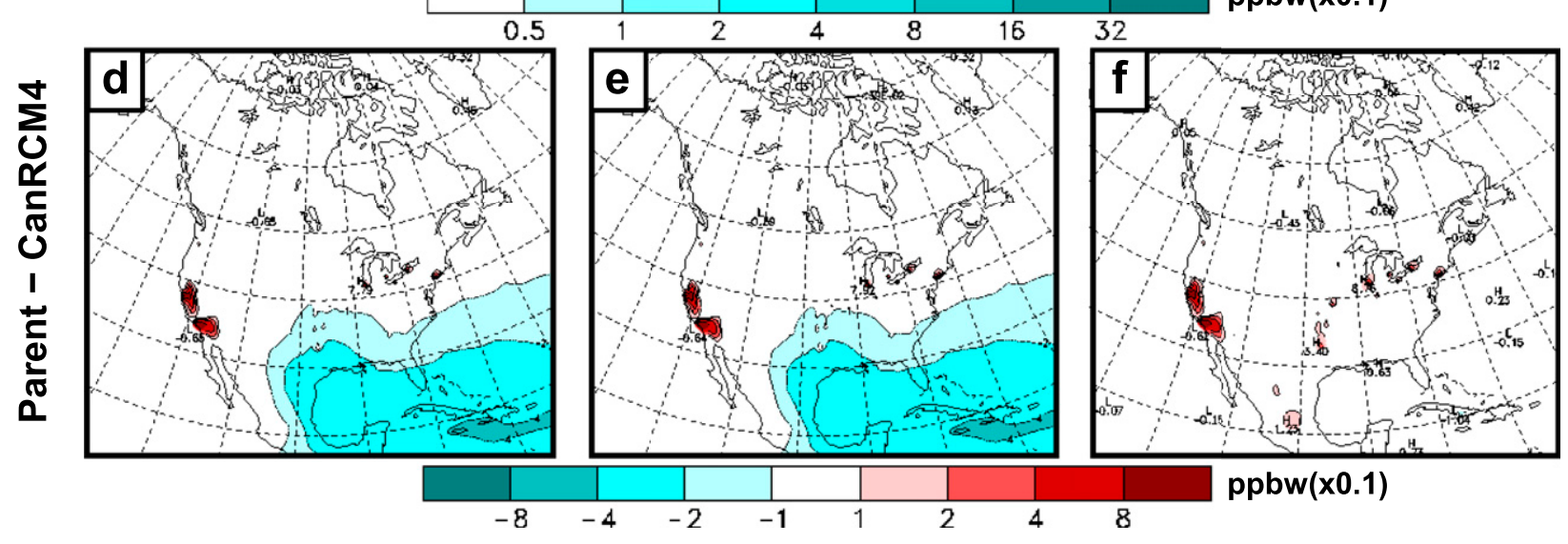

FIG. 2. (top) CanRCM4 mean JJA distribution of near-surface concentration of mineral dust aerosol (2001-05) for the North American domain from three CORDEX evaluation simulations. (a) CanRCM4 is driven by $U, V, T$, and $q$ directly from ERA-Interim data. CanRCM4 is driven by its parent model, which was nudged toward ERA-Interim data employing (b) $U, V, T$, and $q$ and (c) all prognostic variables. (d)-(f) Difference plots of the mineral dust concentration in each of the three CanRCM4 simulations minus that in the nudged simulation of CanRCM4's parent model. In the evaluation of all differences plots, the GCM fields were first interpolated to the finer RCM grid.

region. If such effects are to be downscaled by regional climate models, it is essential that appropriate boundary forcing be employed. By following a coordinated global and regional climate modeling program, a more complete downscaling of climate change effects is possible because of the ability to supply all-field driving of the RCM.

\section{RCM added value in climate projections}

When a regional climate model is used to investigate and understand an issue related to climate change, it is necessarily the case that an understanding of that issue will already be available from the global model simulations that provided the RCM driving data. The downscaling exercise, then, is essentially one of adding understanding, or value, to an existing GCM result. Furthermore, as discussed in section 1 under a coordinated modeling framework, an assessment of value added is essential to justify the resources invested in the development and application of the RCM. Consequently, any statements made regarding the value added by RCM downscaling must be made in the context of GCM results.

While such added understanding is central to the downscaling exercise, its evaluation is an intrinsically difficult undertaking for a variety of reasons, not least of which is the lack of a consensus on how added value should be defined (e.g., see Di Luca et al. 2015). Irrespective of the definition of added value, however, one can still make progress on this issue. Added value can only exist where appreciable differences occur in the climate change results of the global and regional models. The occurrence of appreciable differences represents a necessary but not sufficient condition for the existence of added value. In this section, we develop a framework to define and evaluate appreciable differences between 
ERA-interim Driven $(U, V, T, q)$

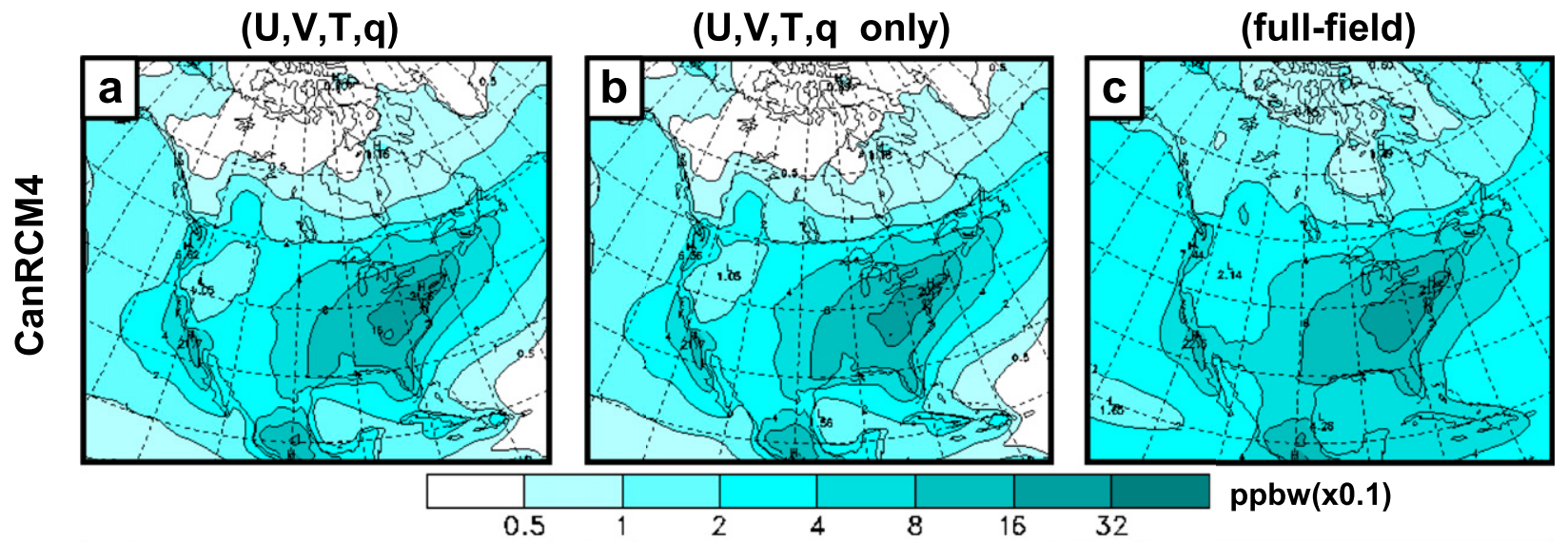

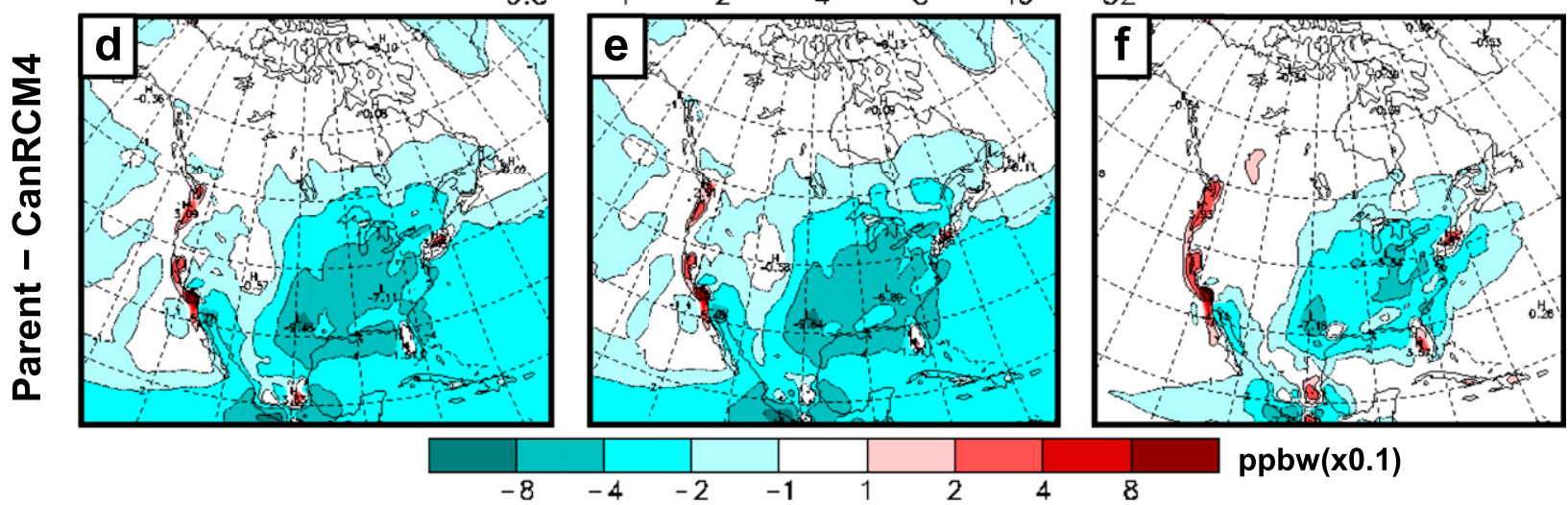

FIG. 3. (top) CanRCM4 mean distribution of near-surface concentration of sulfate aerosol (2001-05) for the North American domain from three CORDEX evaluation simulations. (a) CanRCM4 is driven by $U, V, T$, and $q$ directly from ERA-Interim data. CanRCM4 is driven by its parent model, which was nudged toward ERA-Interim data employing (b) $U, V, T$, and $q$ and (c) all prognostic variables. (d)-(f) Difference plots of the sulfate concentration in CanRCM4 minus that in the nudged simulation of CanRCM4's parent model. In the evaluation of all differences plots, the GCM fields were first interpolated to the finer RCM grid.

GCM and RCM results in the context of climate change problems.

\section{a. Appreciable difference definition}

In a climate projection experiment, the goal is to understand the response of some quantity $X$ to a change in an external forcing of the system. Examples would include the response of precipitation and near-surface temperature to a change in the concentration of greenhouse gases. If resources were unlimited, then an arbitrarily large ensemble of simulations could be performed for a fixed set of climate models to derive an arbitrarily accurate PDF for the change in $X$. Such a response PDF is illustrated in Fig. 4 (top panel). Two response PDFs will exist in this analysis: one for the global model(s) and one for the regional model(s) (bottom panel of Fig. 4).

Given the response PDF, it is then possible to assign a quantitative probability to climate change questions of the following form: What is the probability that the response of $X$ to the external forcing lies between $X_{1}$ and
$X_{2}$ ? The answer is simply given by the shaded area under the response PDF in Fig. 4. For any given climate change question, there will exist a probability for the GCM(s) $\operatorname{Prob}^{\mathrm{GCM}}$ and a probability for the RCM(s) Prob ${ }^{\mathrm{RCM}}$. Since the results of climate projection experiments are primarily expressed in this manner, appreciable differences in the GCM and RCM responses will be taken here to mean appreciable differences in the two probabilities Prob ${ }^{\mathrm{GCM}}$ and Prob $^{\mathrm{RCM}}$. In general, the difference $\Delta$ Prob $=\operatorname{Prob}^{\mathrm{RCM}}-\operatorname{Prob}^{\mathrm{GCM}}$ will never be exactly zero. In practice, a threshold must be set on $\Delta$ Prob to decide if differences are substantive. The specific magnitude of this threshold is not universal as it will depend on the quantity $X$ and the specific application.

The concept of appreciable differences adopted here inherently depends on an application-dependent threshold against which $\Delta$ Prob is evaluated. As such, it is wholly independent of sampling uncertainty, which, for a finite ensemble size, determines whether the two values of 

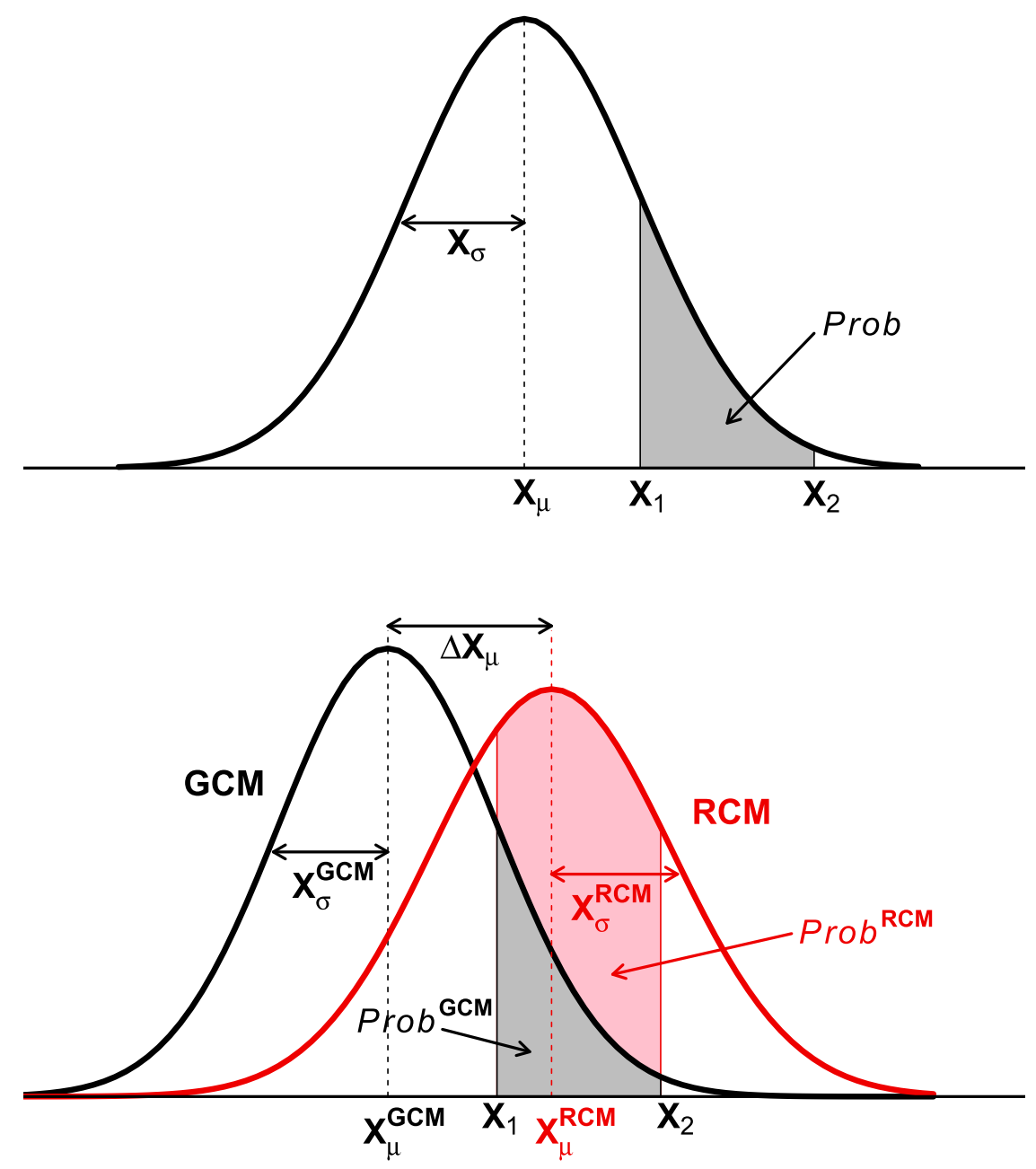

FIG. 4. (top) Illustration of the PDF describing the response of some quantity $X$ to a change in external forcing of the climate system. In practice, such a PDF is constructed from an ensemble of climate change simulations. It allows questions such as, what is the probability that the $X$ response falls within the range $X_{1}$ and $X_{2}$ ? This is provided by the shaded area under the PDF curve in this figure. In this study, the response PDF's properties are characterized by the response mean $X_{\mu}$ and the response spread $X_{\sigma}$. (bottom) Illustration of climate change response PDFs derived from GCM experiments (black) and their corresponding RCM downscaling experiments (red) for $X$.

Prob $^{\mathrm{RCM}}$ and $\mathrm{Prob}^{\mathrm{GCM}}$ are statistically distinguishable. As a consequence, a value of $\Delta$ Prob might be deemed appreciable at some geographic location but not statistically significant and so potentially spurious. In such a case, an increased sample size would be required if a more definitive answer regarding appreciable differences were desired. The impact of sampling uncertainty on the evaluation of appreciable differences will be illustrated in section $5 \mathrm{c}$.

\section{b. Appreciable difference analysis}

In this section, we provide an analysis of the occurrence of appreciable differences in the GCM and RCM results as defined in the previous subsection. The approach taken here is to derive simple, leading-order diagnostics of the RCM and GCM response PDFs that directly characterize differences in the probabilities Prob $^{\mathrm{RCM}}$ and Prob $^{\mathrm{GCM}}$. Leading-order properties of the response PDF are characterized by the response mean $X_{\mu}$ and the response spread $X_{\sigma}$ represented here by the standard deviation (SD). These are illustrated in Fig. 4.

For the purpose of clarity, the analysis will first be described for the case in which the response spread in the RCM is identical to that in the GCM (i.e., $\left.X_{\sigma}^{\mathrm{GCM}} \equiv X_{\sigma}^{\mathrm{RCM}}\right)$. In this instance, a primary measure of the change in the response PDFs is given by the change 
in the response means, $\Delta X_{\mu}=X_{\mu}^{\mathrm{RCM}}-X_{\mu}^{\mathrm{GCM}}$. An obvious question is, how large must this difference be in order for it to cause appreciable differences in the values of Prob ${ }^{\mathrm{GCM}}$ and $\mathrm{Prob}^{\mathrm{RCM}}$ ? It seems sensible to normalize $\Delta X_{\mu}$ by the $\mathrm{SD}\left(X_{\sigma}^{\mathrm{GCM}}\right)$ to produce a standardized difference of the response means:

$$
\widehat{\Delta X_{\mu}}=\Delta X_{\mu} / X_{\sigma}^{\mathrm{GCM}}
$$

as this expresses $\Delta X_{\mu}$ in units of the response spread. Intuitively, one would expect that, when $\left|\overline{\Delta X_{\mu}}\right| \ll 1$, the values of Prob ${ }^{\mathrm{GCM}}$ and $\operatorname{Prob}^{\mathrm{RCM}}$ will be very similar, and little or no appreciable differences should occur.

At leading order, the standardized difference of the response means (4) can be shown to be directly related to the quantity of interest, $\Delta$ Prob $=$ Prob $^{\mathrm{RCM}}-$ Prob $^{\mathrm{GCM}}$. In the appendix, this relationship is derived for the case of a PDF of a Gaussian, or normal, distribution. This example highlights an important point in the comparison of RCM and GCM climate change results. The natural tendency is to analyze the difference of the response means $\Delta X_{\mu}$ between RCM and GCM quantities of interest. In practice, however, an analysis of the standardized difference of the response means provides a more informative diagnostic of the potential impact of RCM downscaling on the probabilistic results derived from GCM climate change experiments (i.e., $\operatorname{Prob}^{\mathrm{GCM}}$ ).

If we relax the assumption $X_{\sigma}^{\mathrm{GCM}} \equiv X_{\sigma}^{\mathrm{RCM}}$, an equivalent leading-order analysis of appreciable differences can be performed in terms of standardized differences of the response spreads:

$$
\widehat{\Delta X_{\sigma}}=\Delta X_{\sigma} / X_{\sigma}^{\mathrm{GCM}},
$$

where $\Delta X_{\sigma}=X_{\sigma}^{\mathrm{RCM}}-X_{\sigma}^{\mathrm{GCM}}$. In the appendix, it is similarly shown that $\widehat{\Delta X_{\sigma}}$ is directly related to $\Delta$ Prob at leading order.

\section{c. Application to CanRCM4 downscaling of CanESM2}

Application of this approach to identify appreciable response differences between CanRCM4 and its parent driving model CanESM2 is provided in Figs. 5-7 for mean precipitation, mean near-surface temperature, and precipitation extremes, respectively. The analysis is derived from an ensemble of five CanESM2 climate change simulations and their corresponding CanRCM4 downscaled counterparts over the CORDEX North American domain employing $0.44^{\circ}$ resolution. For this example, we have used the five-member CanESM2 RCP4.5 contribution to CMIP5 and have augmented the single CanRCM4 downscaling contribution to CORDEX with four additional CanRCM4 simulations driven by the remaining four CanESM2 ensemble members.

In Fig. 5, the March-May ensemble response means of total precipitation $P_{\mu}$, expressed as the percentage change between the two periods 1986-2005 and 2081-2100, are presented for CanESM2 in (Fig. 5a) and CanRCM4 (Fig. 5b). The difference of the response means $\Delta P_{\mu}$ is shown in Fig. 5c. Appreciable response differences of precipitation are diagnosed in Fig. 5d, where the standardized difference of the RCM and GCM response means $\widehat{\Delta P_{\mu}}$ is presented. A comparison of Figs. $5 \mathrm{~d}$ and $5 \mathrm{c}$ indicates that many of the differences in the response means in Fig. $5 c$ are small relative to the CanESM2 ensemble response spread $P_{\mu}^{\mathrm{GCM}}$, suggesting they will have little effect on the resulting climate change probability statements. From Fig. 5d, it can be seen that the largest difference in standardized response means between CanRCM4 and CanESM2 (a precipitation enhancement in the RCM) occurs on the west coast of North America and straddles the border between Canada and the United States. The analysis suggests that this is a viable location to look for value added by the RCM.

As mentioned in section 5a, sampling uncertainty may cause RCM and GCM responses to be indistinguishable even though their difference might exceed the threshold set to be deemed appreciable. The application of a statistical significance test is required to address this possibility. The response differences in Figs. 5e,f are displayed only where they are statistically significant at the $5 \%$ level. Gray shading is used to mask out regions where differences are not statistically significant. These final two panels indicate that, even though differences in much of the domain are not statistically significant, the precipitation response enhancement in the RCM on the west coast of North America remains.

The analysis performed in Fig. 5 for total precipitation response is reproduced in Fig. 6 for near-surface temperature $T$ response. The difference in response means (Fig. 6c) is negative over nearly all of North America. From Fig. 6d, standardized differences are employed to reveal areas of appreciable differences in the RCM and GCM $T$ response, which are mostly statistically significant.

Finally, the appreciable difference analysis performed in Figs. 5 and 6 for time-mean quantities is repeated in Fig. 7 for the 10-yr return value of annual extremes of 24-h precipitation amounts $\mathrm{P} 10$, which is defined as the value that is exceeded by an annual extreme with a probability of $10 \%$. This quantity may also be interpreted as the annual extreme value that is exceeded on average once every $10 \mathrm{yr}$. The 10-yr return values are estimated following the methodology described in Kharin et al. (2013) and references therein. From Figs. 7c,d, it can be seen that differences in the RCM and GCM response means of P10 are 


\section{Precipitation Response}
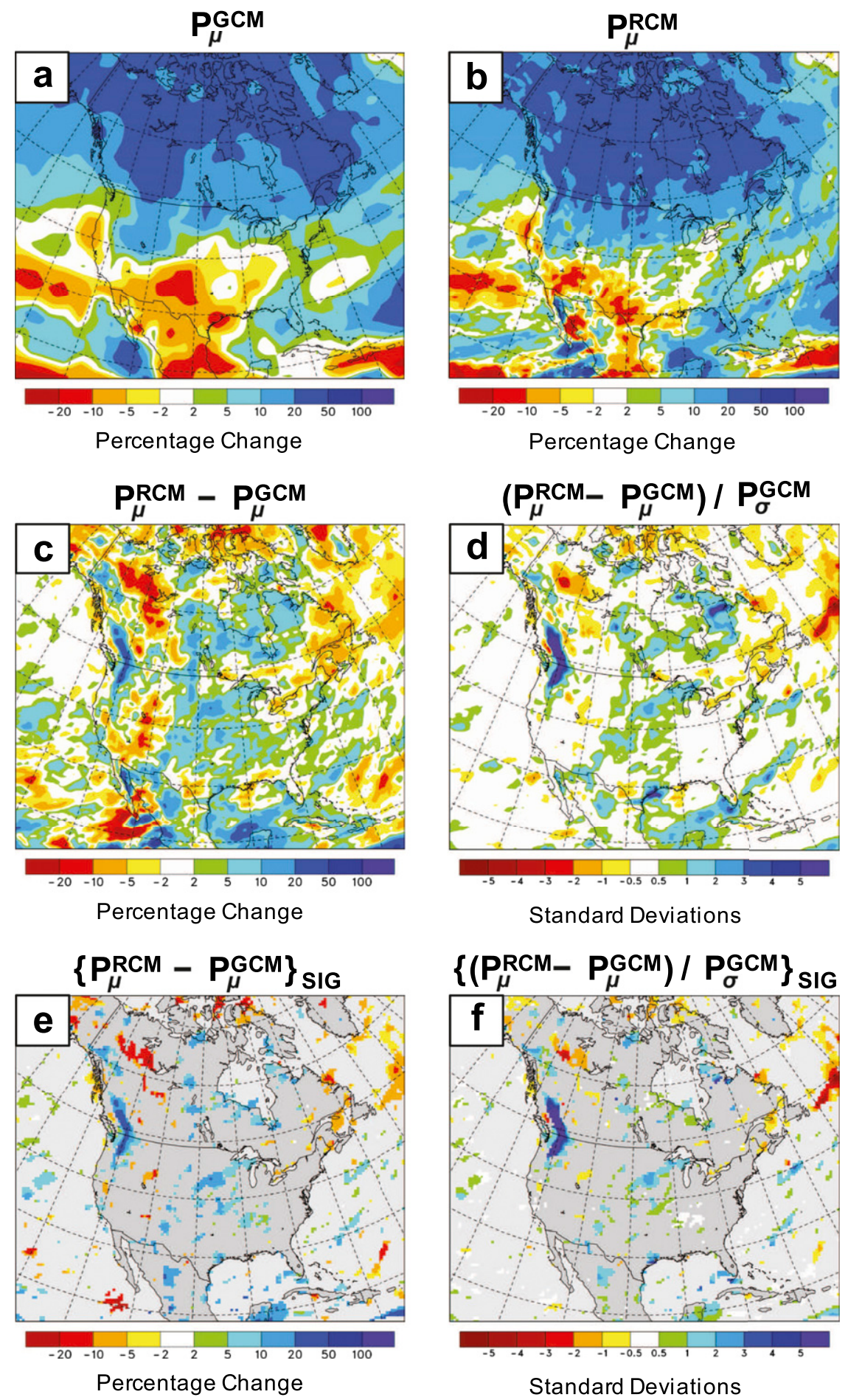

FIG. 5. March-May ensemble response mean of total precipitation expressed as the percentage change between the two periods 1986-2005 and 2081-2100 based on five-member ensembles of (a) CanESM2 and (b) CanRCM4 for the future scenario RCP4.5. Presented are the (c) difference $\Delta P_{\mu}$ and (d) standardized difference $\widehat{\Delta P_{\mu}}$ in the response means. (e),(f) The response differences in (c) and (d) are displayed only where the difference in the response means is statistically significant at the $95 \%$ confidence level. The domain (land) fraction of statistically significant differences is $13 \%(15 \%)$. 


\section{Screen Temperature Response}
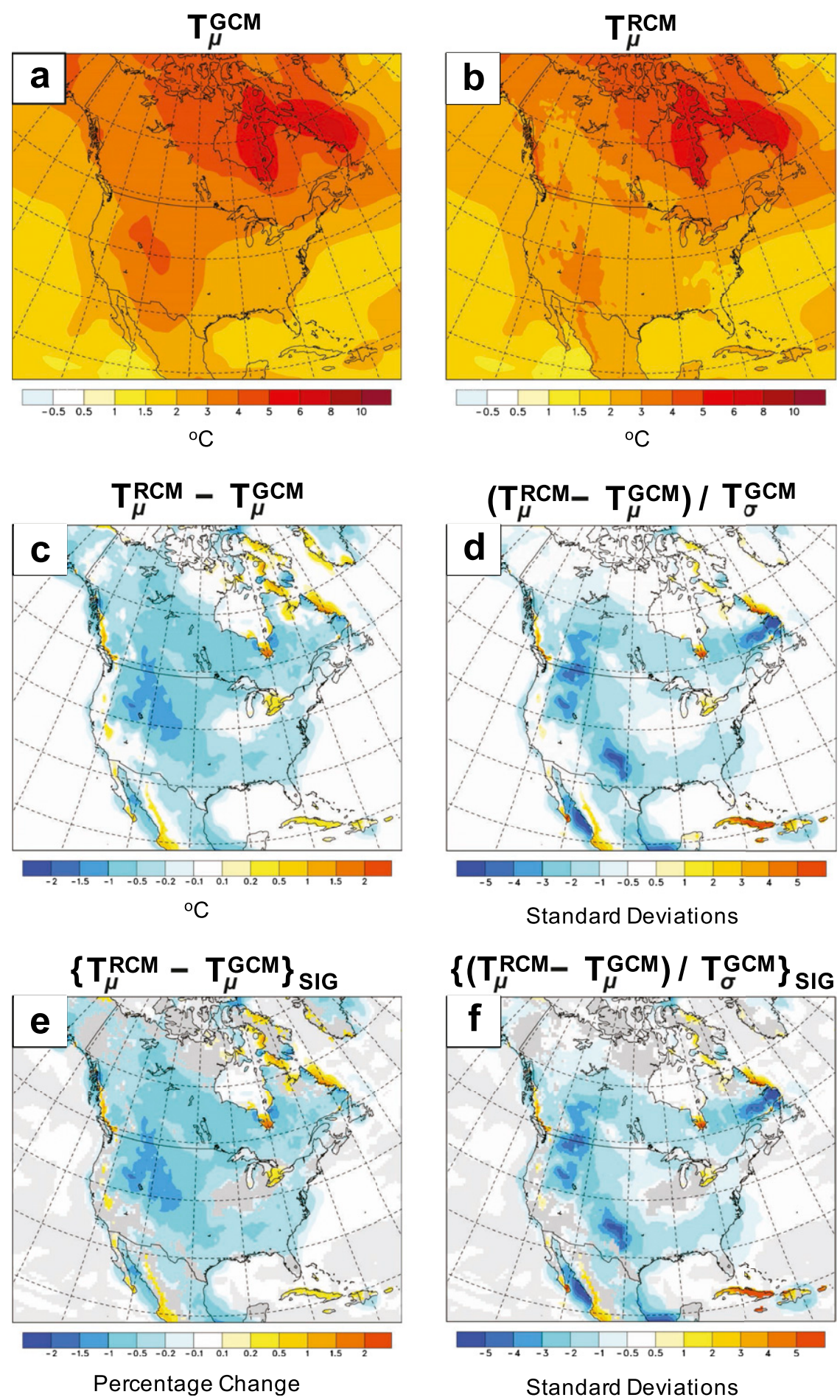

FIG. 6. As in Fig. 5, but for near-surface temperature response $T$. The domain (land) fraction of statistically significant differences is $62 \%(76 \%)$. 


\section{0yr Return Values of Annual Extreme P}
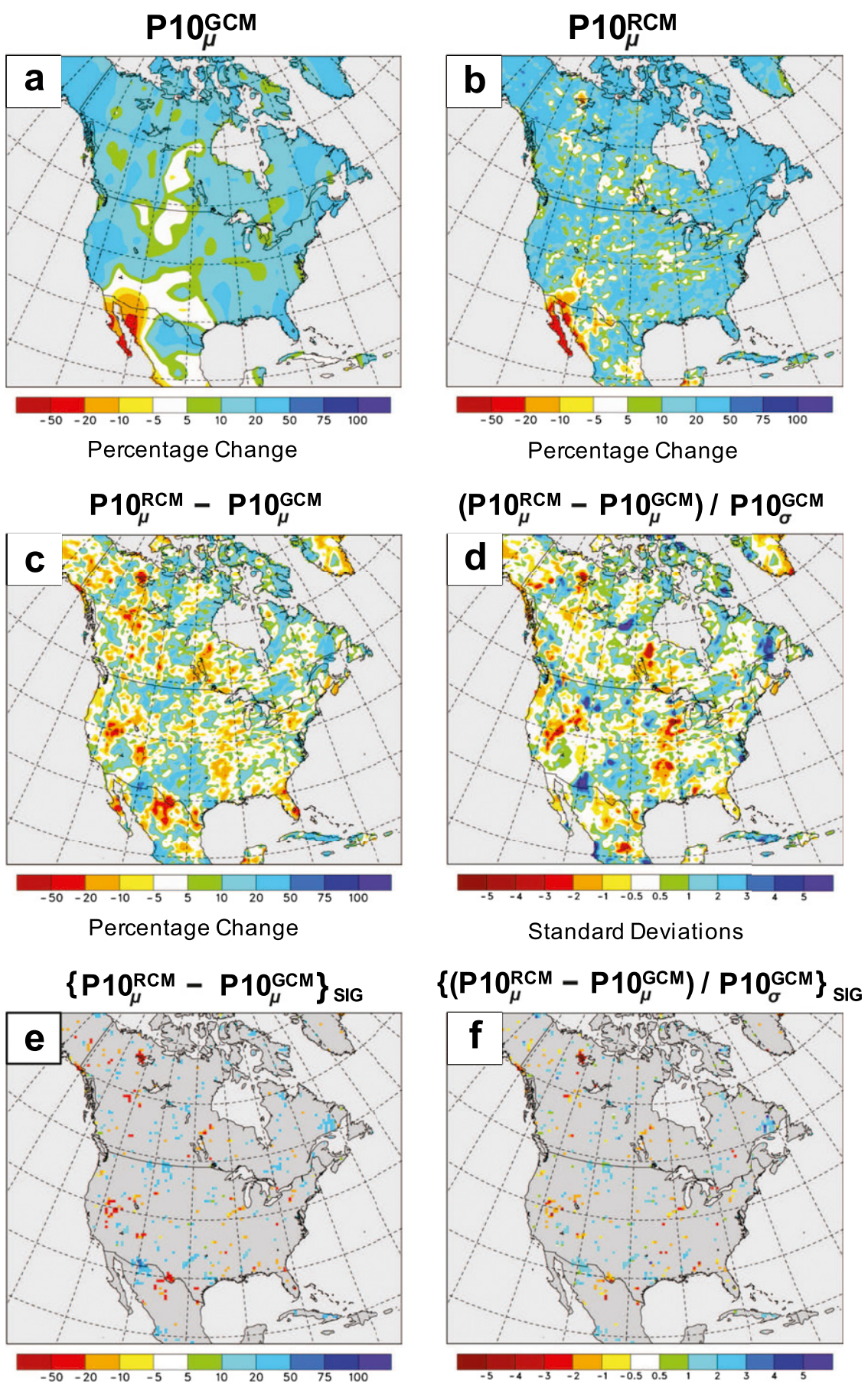

Percentage Change

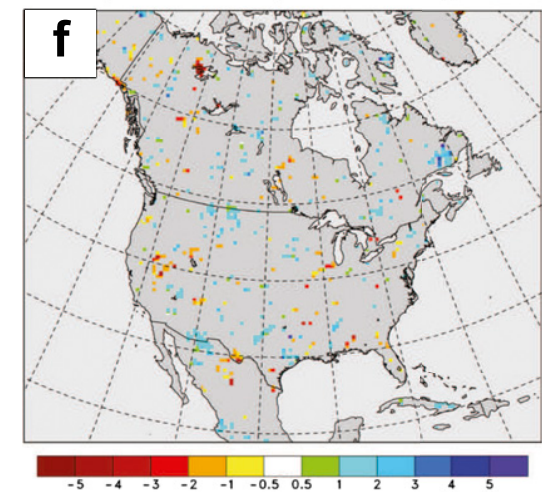

Standard Deviations

FIG. 7. As in Fig. 5, but for the response of 10-yr return values of annual extremes of 24-h precipitation amounts shown over land only. The land fraction of statistically significant differences is $7 \%$. 
fairly noisy relative to those for $P$ and $T$ in Figs. 5 and 6 . Being a higher-order statistic, it is anticipated that P10 might require a larger ensemble than $P$ and $T$ in order to reveal statistically significant differences in the RCM and GCM response means. Figures 7e,f indicate that this does indeed appear to be the case, as only $7 \%$ of the area is covered by statistically significant differences. At the $5 \%$ significance level, it is expected that, on average, $5 \%$ of the area will be found to be statistically significant simply by chance. This highlights the important point that, in the search for added value, higher-order statistics such as extremes may require significantly larger ensemble sizes in order to identify statistically significant appreciable differences in the simulated response of RCMs and GCMs.

\section{d. Discussion}

The preceding application provided an appreciable difference analysis of CanRCM4 and CanESM2 based on their response means. As discussed in section 5b, one can (and likely should) perform the equivalent appreciable difference analysis of the response spread in the RCM and GCM, as this has the potential to affect quantitative probabilistic statements at the same order as appreciable differences in the response means. The present application of the appreciable difference analysis also suggests that higher-order diagnostics, such as extremes, will likely require large ensembles to identify value added by the RCM downscaling - a result that is independent of how one chooses to define added value.

It is also worth noting that response uncertainty derived from a multimodel ensemble represents the overall uncertainty more accurately than response uncertainty derived from a single-model ensemble, such as that presented here. Following the larger vision of the CORDEX project, where ideally many RCMs are used to downscale many CMIP5 GCMs (e.g., Giorgi et al. 2009), standardized changes in the response means and spreads can be defined in terms of the multimodel ensembles, which would constitute a more realistic, and perhaps stringent, criterion for defining appreciable differences.

Finally, in the supplemental material, we repeat the appreciable difference analysis of section $5 b$ for an additional five historical and five future RCP4.5 simulations for a configuration of CanRCM4 in which no spectral nudging is employed. In general, the analysis reveals very similar results as those illustrated in Figs. 5-7. In principle, one could employ the appreciable difference analysis derived here to compare the spectrally nudged and spectrally unnudged RCM climate change results in order to evaluate the impact of upscale influence. As discussed in section 2, novel approaches to analyzing such appreciable differences would need to be developed to determine if such appreciable differences represent an improvement or degradation in either of the configurations.

\section{Summary}

The growing demand for local impacts of future climate change has placed an increasing focus on the regional downscaling of climate change results from global models. At the same time, regional downscaling is one of the more challenging areas of climate change research. As discussed in section 2, the deterministic nature of the current one-way nested approach has an inconsistency that is rooted in the two issues of chaotic divergence and upscale influence. Such inconsistency is the source of potential artifacts that can never be eliminated from downscaling studies of future climate. As a result, the onus is on the modeler to demonstrate that such artifacts have not significantly influenced the results of a downscaling exercise. The best way to accomplish this is currently an open area of research.

In this study, we have presented a program of coordinated global and regional climate modeling, which has been implemented at the Canadian Centre for Climate Modelling and Analysis (CCCma) in the development of its new regional model CanRCM4. It involves a strict adherence to physics compatibility between the RCM and its parent global driving model, CanESM2, and the use of spectral nudging in the development and application of the RCM. As discussed in the body of this paper, the ability to coordinate both global and regional downscaling efforts in this way offers a number of practical and conceptual simplifications that are generally not available to independent RCM modeling centers. For example, CanRCM4 can be driven on its boundaries with all of its prognostic variables, which is critical to getting the correct distribution and future evolution of tracers that have sources outside the regional domain used. Following a two-step procedure in which CanESM is spectrally nudged toward reanalysis (or even another global model), we have shown that CanRCM4 can be driven by its parent model for all applications to realize this benefit. The use of an identical package of physical parameterizations in both the regional and global models also facilitates the development of improved GCM parameterizations and provides a systematic method to investigate their scalability across a range of spatial resolutions. Even so, it is anticipated that, at some point, a physical process may be resolved by the RCM but not the parent GCM, and a parameterization might be present in the latter but not the former. Examples would include deep convection and orographic gravity wave drag. The hope is that the scalability of such 
parameterizations would result in smooth and continuous behavior down to their elimination, but the feasibility of this remains a research topic.

Finally, coordinating global and regional studies of future climate serves to highlight the downscaling exercise as essentially one of adding understanding, or value, to an existing GCM result. A first step in evaluating such added value is the identification of appreciable differences in the climate change results of RCMs and their driving GCMs. In this study, we have provided a framework and shown examples of how the evaluation of such appreciable differences might be formalized and applied to downscaling studies of future climate. An important implication of this approach is that it requires the RCM community to apply its climate change analyses not only to the output of their models but also to the output of the driving GCMs. In order for this to happen, however, the GCM modeling community must provide not only RCM driving data but also satisfy the same data request as that posed to RCM studies of climate change. This was not the case for CMIP5 GCMs, so results of the CORDEX project cannot be generally subjected to the same analysis as that presented here. Consequently, it is recommended that in the future all global models that provide regional model driving data also be required to output overlapping data requests with the regional models so that issues related to value added can begin to be investigated in a more systematic and quantitative manner.

Acknowledgments. The authors are grateful to E. Diaconescu and D. Plummer for their constructive and useful comments made on an earlier version of the manuscript.

\section{APPENDIX}

\section{The Relationship of $\Delta$ Prob to the Standardized Differences $\widehat{\Delta X_{\mu}}$ and $\widehat{\Delta X_{\sigma}}$}

In section 5, appreciable differences have been defined in terms of the quantitative probability difference $\Delta$ Prob. In practice, the probabilities illustrated in Fig. 4 are evaluated from the cumulative distribution function (CDF), $F(x) \equiv \operatorname{Prob}(X \leq x)$, which provides the probability that $X$ is less than $x$. The probability that $X$ lies between $X_{1}$ and $X_{2}$ is simply given by $F\left(X_{1}\right)-F\left(X_{2}\right)$. The $\mathrm{CDF}$ is related to the PDF of section 5 by

$$
\operatorname{PDF}(x) \equiv f(x)=\frac{d F(x)}{d x},
$$

if $F(x)$ is everywhere differentiable or, equivalently, if $f(x)$ is everywhere continuous.
In the analysis presented here, we assume Gaussian, or normal, distributions [i.e., $F(x)=N(x ; \mu, \sigma)$ ], which allows us to write

$$
\begin{aligned}
\Delta \operatorname{Prob} & =\operatorname{Prob}^{\mathrm{RCM}}-\operatorname{Prob}^{\mathrm{GCM}} \\
& \equiv \Delta \operatorname{Prob}\left(X_{2}\right)-\Delta \operatorname{Prob}\left(X_{1}\right),
\end{aligned}
$$

where

$$
\begin{aligned}
\Delta \operatorname{Prob}\left(X_{i}\right) & =N\left(X_{i} ; X_{\mu}^{\mathrm{RCM}}, X_{\sigma}^{\mathrm{RCM}}\right)-N\left(X_{i} ; X_{\mu}^{\mathrm{GCM}}, X_{\sigma}^{\mathrm{GCM}}\right) \\
& =N\left(\frac{X_{i}-X_{\mu}^{\mathrm{RCM}}}{X_{\sigma}^{\mathrm{RCM}}} ; 0,1\right)-N\left(\frac{X_{i}-X_{\mu}^{\mathrm{GCM}}}{X_{\sigma}^{\mathrm{GCM}}} ; 0,1\right) \\
& =\hat{N}\left(Z_{i}^{\mathrm{RCM}}\right)-\hat{N}\left(Z_{i}^{\mathrm{GCM}}\right),
\end{aligned}
$$

where (A4) results from a transform to the standard normal distribution $\hat{N}$ (i.e., that with zero mean and unity standard deviation), and $Z_{i} \equiv\left(X_{i}-X_{\mu}\right) / X_{\sigma}$.

As described in section 5, prior to any RCM downscaling of future projections, there will exist a series of GCM simulations for the equivalent experiment and a set of quantitative probabilities $\mathrm{Prob}^{\mathrm{GCM}}$ for a range of climate change questions. The appreciable difference analysis poses the following question: How might these GCM derived probabilities be altered by the results of the RCM downscaling? From this perspective, the magnitude of $\Delta$ Prob will depend only on the two quantities $X_{\mu}^{\mathrm{RCM}}$ and $X_{\sigma}^{\mathrm{RCM}}$ in (A3), as the remaining quantities in this expression are all known prior to application of the RCM. Rewriting $Z_{i}^{\mathrm{RCM}}$ as

$$
\begin{aligned}
Z_{i}^{\mathrm{RCM}} & \equiv \frac{X_{i}-X_{\mu}^{\mathrm{RCM}}}{X_{\sigma}^{\mathrm{RCM}}}=\frac{\left(X_{i}-X_{\mu}^{\mathrm{GCM}}\right) / X_{\sigma}^{\mathrm{GCM}}-\widehat{\Delta X_{\mu}}}{1+\widehat{\Delta X_{\sigma}}} \\
& =\frac{Z_{i}^{\mathrm{GCM}}-\widehat{\Delta X_{\mu}}}{1+\widehat{\Delta X_{\sigma}}},
\end{aligned}
$$

allows this dependence of $\Delta$ Prob to be equivalently expressed in terms of the standardized difference of the response means $\widehat{\Delta X_{\mu}}$ and the standardized difference of the response spreads $\widehat{\Delta X_{\sigma}}$.

Finally, assuming $\left|\widehat{\Delta X_{\mu}}\right| \ll 1$ and $\left|\widehat{\Delta X_{\sigma}}\right| \ll 1$, a Taylor expansion of the function $\Delta \operatorname{Prob}\left(\widehat{\Delta X_{\mu}}, \widehat{\Delta X_{\sigma}} ; Z_{i}^{\mathrm{GCM}}\right)$ may be performed about the zero value of $\widehat{\Delta X_{\mu}}$ and $\widehat{\Delta X_{\sigma}}$ as

$$
\begin{aligned}
\Delta \operatorname{Prob}= & {\left[f_{\hat{N}}\left(Z_{1}^{\mathrm{GCM}}\right)-f_{\hat{N}}\left(Z_{2}^{\mathrm{GCM}}\right)\right] \widehat{\Delta X_{\mu}}+\left[f_{\hat{N}}\left(Z_{1}^{\mathrm{GCM}}\right) Z_{1}^{\mathrm{GCM}}\right.} \\
& \left.-f_{\hat{N}}\left(Z_{2}^{\mathrm{GCM}}\right) Z_{2}^{\mathrm{GCM}}\right] \widehat{\Delta X_{\sigma}}+O(2),
\end{aligned}
$$


where $f_{\hat{N}}$ is the PDF (A1) of the standard normal distribution, demonstrating that $\Delta$ Prob is proportional to $\widehat{\Delta X_{\mu}}$ and $\widehat{\Delta X_{\sigma}}$ at leading order.

\section{REFERENCES}

Abdella, K., and N. A. McFarlane, 1996: Parameterization of the surface-layer exchange coefficients for atmospheric models. Bound.-Layer Meteor., 80, 223-248, doi:10.1007/ BF00119544.

Adler, R., and Coauthors, 2003: The Version-2 Global Precipitation Climatology Project (GPCP) monthly precipitation analysis (1979-present). J. Hydrometeor., 4, 1147-1167, doi:10.1175/ 1525-7541(2003)004<1147:TVGPCP > 2.0.CO;2.

Alexandru, A., R. de Elía, R. Laprise, L. Separovic, and S. Biner, 2009: Sensitivity study of regional climate model simulations to large-scale nudging parameters. Mon. Wea. Rev., 137, 16661686, doi:10.1175/2008MWR2620.1.

Arora, V. K., and Coauthors, 2011: Carbon emission limits required to satisfy future representative concentration pathways of greenhouse gases. Geophys. Res. Lett., 38, L05805, doi:10.1029/ 2010GL046270.

Barker, H. W., J. N. S. Cole, J. J. Morcrette, R. Pincus, P. Raisanen, K. von Salzen, and P. A. Vaillancourt, 2008: The Monte Carlo independent column approximation: An assessment using several global atmospheric models. Quart. J. Roy. Meteor. Soc., 134, 1463-1478, doi:10.1002/qj.303.

Biner, S., D. Caya, R. Laprise, and L. Spacek, 2000: Nesting of RCMs by imposing large scales. Research activities in atmospheric and oceanic modelling. WMO/TD-987 30, 7.3-7.4.

Bowden, J. H., C. G. Nolte, and T. L. Otte, 2013: Simulating the impact of the large-scale circulation on the 2-m temperature and precipitation climatology. Climate Dyn., 40, 1903-1920, doi:10.1007/s00382-012-1440-y.

Castro, C. L., R. A. Pielke, and G. Leoncini, 2005: Dynamical downscaling: Assessment of value retained and added using the Regional Atmospheric Modeling System (RAMS). J. Geophys. Res., 110, D05108, doi:10.1029/2004JD004721.

Chaboureau, J. P., and P. Bechtold, 2005: Statistical representation of clouds in a regional model and the impact on the diurnal cycle of convection during tropical convection, cirrus and nitrogen oxides (TROCCINOX). J. Geophys. Res., 110, D17103, doi:10.1029/2004JD005645.

Côté, J., S. Gravel, A. Methot, A. Patoine, M. Roch, and A. Staniforth, 1998: The operational CMC-MRB Global Environmental Multiscale (GEM) model. Part I: Design considerations and formulation. Mon. Wea. Rev., 126, 1373-1395, doi:10.1175/ 1520-0493(1998)126<1373:TOCMGE > 2.0.CO;2.

Croft, B., U. Lohmann, and K. von Salzen, 2005: Black carbon ageing in the Canadian Centre for Climate Modelling and Analysis atmospheric general circulation model. Atmos. Chem. Phys., 5, 1931-1949, doi:10.5194/acp-5-1931-2005.

Davies, H. C., 1976: A lateral boundary formulation for multi-level prediction models. Quart. J. Roy. Meteor. Soc., 102, 405-418, doi:10.1002/qj.49710243210.

Dee, D. P., and Coauthors, 2011: The ERA-Interim reanalysis: Configuration and performance of the data assimilation system. Quart. J. Roy. Meteor. Soc., 137, 553-597, doi:10.1002/qj.828.

Denis, B., J. Côté, and R. Laprise, 2002: Spectral decomposition of two-dimensional atmospheric fields on limited-area domains using the discrete cosine transform (DCT). Mon. Wea. Rev., 130, 1812-1829, doi:10.1175/1520-0493(2002)130<1812: SDOTDA $>2.0 . \mathrm{CO} ; 2$.
Di Luca, A., R. de Ela, and R. Laprise, 2015: Challenges in the quest for added value of regional climate dynamical downscaling. Current Climate Change Rep., 1, 10-21, doi:10.1007/ s40641-015-0003-9.

Engelstaedter, S., I. Tegen, and R. Washington, 2006: North African dust emissions and transport. Earth-Sci. Rev., 79, 73-100, doi:10.1016/j.earscirev.2006.06.004.

Feser, F., and M. Barcikowska, 2012: The influence of spectral nudging on typhoon formation in regional climate models. Environ. Res. Lett., 7, 014024, doi:10.1088/1748-9326/7/1/ 014024.

Giorgi, F., 2006: Regional climate modeling: Status and perspectives. J. Phys., 139, 101-118, doi:10.1051/jp4:2006139008.

— Regional climate modeling revisited. J. Geophys. Res., 104, 6335-6352, doi:10.1029/98JD02072.

_ C. Jones, and G. Asrar, 2009: Addressing climate information needs at the regional level: The CORDEX framework. WMO Bull., 58, 175-183.

Glisan, J. M., W. J. Gutowski, J. J. Cassano, and M. E. Higgins, 2013: Effects of spectral nudging in WRF on Arctic temperature and precipitation simulations. J. Climate, 26, 3985-3999, doi:10.1175/JCLI-D-12-00318.1.

Griffin, D. W., 2007: Atmospheric movement of microorganisms in clouds of desert dust and implications for human health. Clin. Microbiol. Rev., 20, 459-477, doi:10.1128/CMR.00039-06.

Hernandez-Diaz, L., R. Laprise, L. Sushama, A. Martynov, K. Winger, and B. Dugas, 2012: Climate simulation over CORDEX Africa domain using the fifth-generation Canadian Regional Climate Model (CRCM5). Climate Dyn., 40, 14151433, doi:10.1007/s00382-012-1387-z.

Jacob, D., and Coauthors, 2012: Assessing the transferability of the regional climate model REMO to different Coordinated Regional Climate Downscaling Experiment (CORDEX) regions. Atmosphere, 3, 181-199, doi:10.3390/atmos3010181.

Kanamaru, H., and M. Kanamitsu, 2007: Scale-selective bias correction in a downscaling of global analysis using a regional model. Mon. Wea. Rev., 135, 334-350, doi:10.1175/ MWR3294.1.

Khairoutdinov, M., and Y. Kogan, 2000: A new cloud physics parameterization in a large-eddy simulation model of marine stratocumulus. Mon. Wea. Rev., 128, 229-243, doi:10.1175/ 1520-0493(2000)128<0229:ANCPPI > 2.0.CO;2.

Kharin, V. V., and J. F. Scinocca, 2012: The impact of model fidelity on seasonal predictive skill. Geophys. Res. Lett., 39, L18803, doi:10.1029/2012GL052815.

—, F. Zwiers, X. Zhang, and M. Wehner, 2013: Changes in temperature and precipitation extremes in the CMIP5 ensemble. Climatic Change, 119, 345-357, doi:10.1007/ s10584-013-0705-8.

Koltzow, M., T. Iversen, and J. E. Haugen, 2008: Extended BigBrother experiments: The role of lateral boundary data quality and size of integration domain in regional climate modelling. Tellus, 60A, 398-410, doi:10.1111/j.1600-0870.2008.00309.x.

Laprise, R., 2008: Regional climate modelling. J. Comput. Phys., 227, 3641-3666, doi:10.1016/j.jcp.2006.10.024.

Li, J., 2002: Accounting for unresolved clouds in a 1D infrared radiative transfer model. Part I: Solution for radiative transfer, including cloud scattering and overlap. J. Atmos. Sci., 59, 3302-3320, doi:10.1175/1520-0469(2002)059<3302:AFUCIA >2.0.CO;2.

_ correlated- $k$ distribution. Part I: Local thermal equilibrium. J. Atmos. Sci., 62, 286-309, doi:10.1175/JAS-3396.1. 
Lohmann, U., and E. Roeckner, 1996: Design and performance of a new cloud microphysics scheme developed for the ECHAM general circulation model. Climate Dyn., 12, 557-572, doi:10.1007/ BF00207939.

_ , K. von Salzen, N. McFarlane, H. G. Leighton, and J. Feichter, 1999: Tropospheric sulfur cycle in the Canadian general circulation model. J. Geophys. Res., 104, 26 833-26 858, doi:10.1029/ 1999JD900343.

Lucas-Picher, P., D. Caya, S. Biner, and R. Larpise, 2008a: Quantification of the lateral boundary forcing of a regional climate model using an aging tracer. Mon. Wea. Rev., 136, 4980-4996, doi:10.1175/2008MWR2448.1.

,,-- R. de Elía, and R. Larpise, 2008b: Investigation of regional climate models' internal variability with a ten-member ensemble of 10-year simulations over a large domain. Climate Dyn., 31, 927-940, doi:10.1007/s00382-008-0384-8.

McFarlane, N. A., J. F. Scinocca, M. Lazare, R. Harvey, D. Verseghy, and J. Li, 2006: The CCCma third generation Atmospheric General Circulation Model (AGCM3). CCma Internal Tech. Rep., 28 pp. [Available online at ftp://ftp. cccma.ec.gc.ca/pub/jscinocca/reports/AGCM3_report.pdf.]

Mearns, L. O., W. J. Gutowski, R. Jones, L. Y. Leung, S. McGinnis, A. M. B. Nunes, and Y. Qian, 2009: A regional climate change assessment program for North America. Eos, Trans. Amer. Geophys. Union, 90, 311, doi:10.1029/2009EO360002.

— , and Coauthors, 2012: The North American Regional Climate Change Assessment Program: Overview of phase I results. Bull. Amer. Meteor. Soc., 93, 1337-1362, doi:10.1175/ BAMS-D-11-00223.1.

Miguez-Macho, G., G. L. Stenchikov, and A. Robock, 2004: Spectral nudging to eliminate the effects of domain position and geometry in regional climate model simulations. J. Geophys. Res., 109, 693-712, doi:10.1029/2003JD004495.

Minnis, P., D. Young, S. Sun-Mack, P. W. Heck, D. R. Doelling, and Q. Z. Trepte, 2003: CERES cloud property retrievals from imagers on TRMM, Terra, and Aqua. Remote Sensing of Clouds and the Atmosphere VIII, K. P. Schaefer et al., Eds., International Society for Optical Engineering (SPIE Proceedings, Vol. 5235), 37-48, doi:10.1117/12.511210.

Otte, T. L., C. G. Nolte, M. J. Otte, and J. H. Bowden, 2012: Does nudging squelch the extremes in regional climate modeling? J. Climate, 25, 7046-7066, doi:10.1175/JCLI-D-12-00048.1.

Pincus, R., H. W. Barker, and J. J. Morcrette, 2003: A fast, flexible, approximate technique for computing radiative transfer in inhomogeneous cloud fields. J. Geophys. Res., 108, 4376, doi:10.1029/ 2002JD003322.

Rockel, B., C. L. Castro, R. A. Pielke, H. von Storch, and G. Leoncini, 2008: Dynamical downscaling: Assessment of model system dependent retained and added variability for two different regional climate models. J. Geophys. Res., 113, D21107, doi:10.1029/2007JD009461.
Rotstayn, L. D., 1997: A physically based scheme for the treatment of stratiform clouds and precipitation in large-scale models. I: Description and evaluation of the microphysical processes. Quart. J. Roy. Meteor. Soc., 123, 1227-1282, doi:10.1002/ qj. 49712354106.

Scinocca, J. F., N. A. McFarlane, M. Lazare, J. Li, and D. Plummer, 2008: Technical Note: The CCCma third generation AGCM and its extension into the middle atmosphere. Atmos. Chem. Phys., 8, 7055-7074, doi:10.5194/acp-8-7055-2008.

Song, S., J. Tang, and X. Chen, 2011: Impacts of spectral nudging on the sensitivity of a regional climate model to convective parameterizations in East Asia. Acta Meteor. Sin., 25, 63-77, doi:10.1007/s13351-011-0005-z.

Takle, E. S., J. Roads, B. Rockel, W. J. Gutowski, R. W. Arritt, I. Meinke, C. G. Jones, and A. Zadra, 2007: Transferability intercomparison: An opportunity for new insight on the global water cycle and energy budget. Bull. Amer. Meteor. Soc., 88, 375-384, doi:10.1175/BAMS-88-3-375.

Tang, J., S. Song, and J. Wu, 2010: Impacts of the spectral nudging technique on simulation of the East Asian summer monsoon. Theor. Appl. Climatol., 101, 41-51, doi:10.1007/ s00704-009-0202-1.

von Salzen, K., and Coauthors, 2000: Sensitivity of sulphate aerosol size distributions and $\mathrm{CCN}$ concentrations over North America to SOx emissions and $\mathrm{H}_{2} \mathrm{O}_{2}$ concentrations. J. Geophys. Res., 105, 9741-9765, doi:10.1029/2000JD900027.

, N. A. McFarlane, and M. Lazare, 2005: The role of shallow convection in the water and energy cycles of the atmosphere. Climate Dyn., 25, 671-688, doi:10.1007/ s00382-005-0051-2.

— mospheric Global Climate Model (CanAM4). Part I: Representation of physical processes. Atmos.-Ocean, 51, 104-125, doi:10.1080/07055900.2012.755610.

von Storch, H., H. Langenberg, and F. Feser, 2000: A spectral nudging technique for dynamical downscaling purposes. Mon. Wea. Rev., 128, 3664-3673, doi:10.1175/1520-0493(2000)128<3664: ASNTFD $>2.0 . C O ; 2$.

Wielicki, B. A., B. R. Barkstrom, E. F. Harrison, R. B. Lee, G. L. Smith, and J. E. Cooper, 1996: Clouds and the Earth's Radiant Energy System (CERES): An Earth Observing System experiment. Bull. Amer. Meteor. Soc., 77, 853-868, doi:10.1175/ 1520-0477(1996)077<0853:CATERE $>2.0 . C O ; 2$.

Yeh, K. S., J. Côté, S. Gravel, A. Methot, A. Patoine, M. Roch, and A. Staniforth, 2002: The CMC-MRB Global Environmental Multiscale (GEM) model. Part III: Nonhydrostatic formulation. Mon. Wea. Rev., 130, 339-356, doi:10.1175/ 1520-0493(2002)130<0339:TCMGEM > 2.0.CO;2.

Zadra, A., D. Caya, J. Côté, B. Dugas, C. Jones, R. Laprise, K. Winger, and L. P. Caron, 2008: The next Canadian Regional Climate Model. Phys. Canada, 64, 75-83. 